studies only, no heterogeneity was evident in the unadjusted study results. Patients with VRE had stayed in the hospital much longer than control patients. Studies that adjusted for this difference found only a small and nonsignificant association between vancomycin treatment and VRE (pooled OR, 1.4; $\mathrm{CI}_{95}, 0.74-2.60$ ). They also detected publication bias, favoring report of studies that found a large measure of association.

The authors concluded that the reported strong association between vancomycin treatment and hospitalacquired VRE is due to the selection of the reference group and is confounded by duration of hospitalization and publication bias. Studies that accounted for these factors found only a small and nonsignificant association.

FROM: Carmeli Y, Samore MH, Huskins C. The association between antecedent vancomycin treatment and hospital-acquired vancomycin-resistant enterococci: a meta-analysis. Arch Intern Med 1999;159:2461-2468.

\section{Bacterial Resistance to Disinfectants: An Update}

Professor A.D. Russell from the Welsh School of Pharmacy, Cardiff University, recently reviewed the subject of bacterial resistance to germicides. He points out that attention increasingly is being directed to the responses of various types of microbes to biocides (antiseptics, disinfectants, and preservatives). Different groups of bacteria vary in their susceptibility to biocides, with bacterial spores being the most resistant, followed by mycobacteria, then gram-negative organisms, with cocci generally being the most sensitive. There are wide divergences within this general classification. Thus, (1) spores of Bacillus subtilis are less susceptible to biocides than those of Clostridium difficile; (2) Mycobacterium chelonae strains may show high resistance to glutaraldehyde, and Mycobacterium avium intracellulare is generally less sensitive than Mycobacterium tuberculosis; (3) gram-negative bacteria such as Pseudomonas aeruginosa, Providencia species, and Proteus species may be difficult to inactivate; (4) enterococci are less sensitive than staphylococci to biocides, and antibiotic-resistant strains of Staphylococcus aureus might show low-level biocide resistance.

The mechanisms involved in biocide resistance to biocides are becoming understood better. Intrinsic resistance (intrinsic insusceptibility) is found with bacterial spores, mycobacteria, and gram-negative bacteria. This resistance might, in some instances, be associated with constitutive degradative enzymes, but in reality is more closely linked to cellular impermeability. The coat(s) and, to some extent, the cortex in spores, the arabinogalactan, and possibly other components of the mycobacterial cell wall and the outer membrane of gram-negative bacteria limit the concentration of active biocide that can reach the target site(s) in these bacterial cells.

A special situation is found with bacteria present in biofilms, which can be considered as being an intrinsic resistance mechanism resulting from physiological (phenotypic) adaptation of cells. Acquired resistance to biocides may arise by cellular mutation or by the acquisition of genetic elements. Plasmid- and transposon-mediated resistance to inorganic and organic mercury compounds by hydrolases and reductases has been extensively studied. Plasmid-mediated resistance to some other biocides in gram-negative bacteria and in staphylococci has been described, but its significance remains uncertain.

There is a need to establish conclusively whether there is a clear-cut link between antibiotic and biocide resistance in non-sporulating bacteria and whether biocides can select for antibiotic resistance. The responses to biocides of new and emerging pathogens must be assessed. Continuing research is necessary to establish further the underlying mechanisms of resistance and to provide more efficient means of bacterial inactivation.

FROM: Russell AD. Bacterial resistance to disinfectants: present knowledge and future problems. $J$ Hosp Infect 1999;43(suppl):S57-S68. 